1. Bentivoglio M, Pacini P. Filippo Pacini-a determined observer. Brain Res Bull 1995; 38: 161-5.

2. Cobo R, García-Piqueras J, Cobo J, Vega JA. The human cutaneous sensory corpuscles: an update. J Clin Med 2021; 10: 227.

3. Kettenmann H. Nervous system. In: Standring S, editor. Gray's Anatomy: The Anatomical Basis of Clinical Practice. 41st ed. Amsterdam: Elsevier, 2016; 61

4. Landon DN, Wiseman OJ. A Pacinian corpuscle in the human bladder lamina propria. J Neurocytol 2001; 30: 457-64.

5. Standop J, Ulrich A, Schneider MB, Andrén-Sandberg A, Pour PM. Pacinian corpuscle in the human pancreas. Pancreas 2001; 23: 36-9.

6. Aydin O. Pacinian corpuscle in a lymph node. Neuropathology 2006 26: $379-81$.

7. Varga I, Nosál M, Babál P. Ectopic lamellar Pacinian corpuscle within the thymus. Atypical or abnormal location? Rom J Morphol Embryol 2020; 61: 273-6.

8. Gessi M, Messing-Jünger M, Röhrig A, Gielen GH, Pietsch T, van Landeghem FK. Incidental neuropathological findings in a child with cervical meningoradiculocele. Brain Pathol 2012; 22: 737-40.

9. Feito J, Ramos-García JL, Gago Á, et al. Pacinian corpuscles in a cervical chondrocutaneous remnant: a case report and update of Pacinian corpuscles. Am J Dermatopathol 2016; 38: 231-5.

10. Pai SA. Ectopic Pacinian corpuscle in the prostate. Int J Surg Patho 2017: 25: 609-10.

11. Medlicott SAC, Larsen ET, Gao Y, Trpkov K. Pacinian corpuscle in the prostate: fact - not fiction! Hum Pathol Case Rep 2019; 15: 71-2.

12. Dixon JS, Gosling JA, Canning DA, Gearhart JP. An immunohistochemical study of human postnatal paraganglia associated with the urinary bladder. J Anat 1992; 181: 431-6.

DOI: https://doi.org/10.1016/j.pathol.2021.06.126

\section{An unusual testicular mass: a case of desmoplastic small round cell tumour}

To the Editor,

Desmoplastic small round cell tumour (DSRCT) is a rare, aggressive, mesenchymal malignancy of uncertain differentiation with both a characteristic chromosomal translocation and immunohistochemical profile. It most commonly presents with vague abdominal symptoms, most often in young, Caucasian males. Advanced disease on diagnosis with multiple intra-abdominal lesions is common. ${ }^{1}$ While typically an intra-abdominal malignancy, cases of DSRCT arising from lung, central nervous system, nasal sinus and paratesticular lesions have been reported rarely. ${ }^{2-4}$ Presentation as a primary testicular mass is exceedingly rare and poses a diagnostic challenge both in the clinical and pathological setting, as this tumour can mimic many other entities.

We present a case of a 29-year-old, otherwise well, male who presented with an enlarging right scrotal mass. An ultrasound demonstrated an abnormal, hypoechoic right testis measuring $6 \mathrm{~cm}$, concerning for a malignant mass replacing the testis. Unfortunately, he was non-compliant with medical advice and did not attend follow up for 3 months. Eventually, he attended an urgent urology appointment, and a computed tomography scan of the chest, abdomen and pelvis was performed. Imaging demonstrated multiple metastases including pulmonary lesions and diffuse lymphadenopathy in the left supraclavicular fossa, mediastinum and para-aortic regions measuring up to $6 \mathrm{~cm}$. Serum tumour markers were negative. Urgent admission to hospital was arranged, however the patient did not attend despite multiple attempts to facilitate treatment. Two months later, the patient presented to the emergency department requesting treatment. The suspected diagnosis was a testicular germ cell tumour with metastatic disease. Approximately $30 \%$ of patients with testis cancer have metastases at diagnosis.

Radical orchidectomy was performed and the patient recovered uneventfully post-operatively. Macroscopic examination showed a $75 \mathrm{~mm}$ testicular mass with tumour replacing the entire contents of the testis and epididymis (Fig. 1). The tumour was thoroughly sampled to reveal no gross or microscopic heterologous elements, suggestive of a postpubertal-type teratoma. Histologically, this tumour was composed of nests of malignant tumour surrounded by a desmoplastic stroma. The tumour cells had minimal cytoplasm and rounded to oval nuclei. Numerous mitoses were present with focal areas of single cell necrosis. There was extensive lymphovascular invasion with tumour deposits seen within the tunica vaginalis (Fig. 2). The cord contained continuous tumour that was present at the spermatic cord resection margin. Immunohistochemistry showed multiphenotypic differentiation with staining for epithelial membrane antigen (EMA), pancytokeratin (MNF-116), desmin (dot-like cytoplasmic), CD57, CD56 and neuron specific
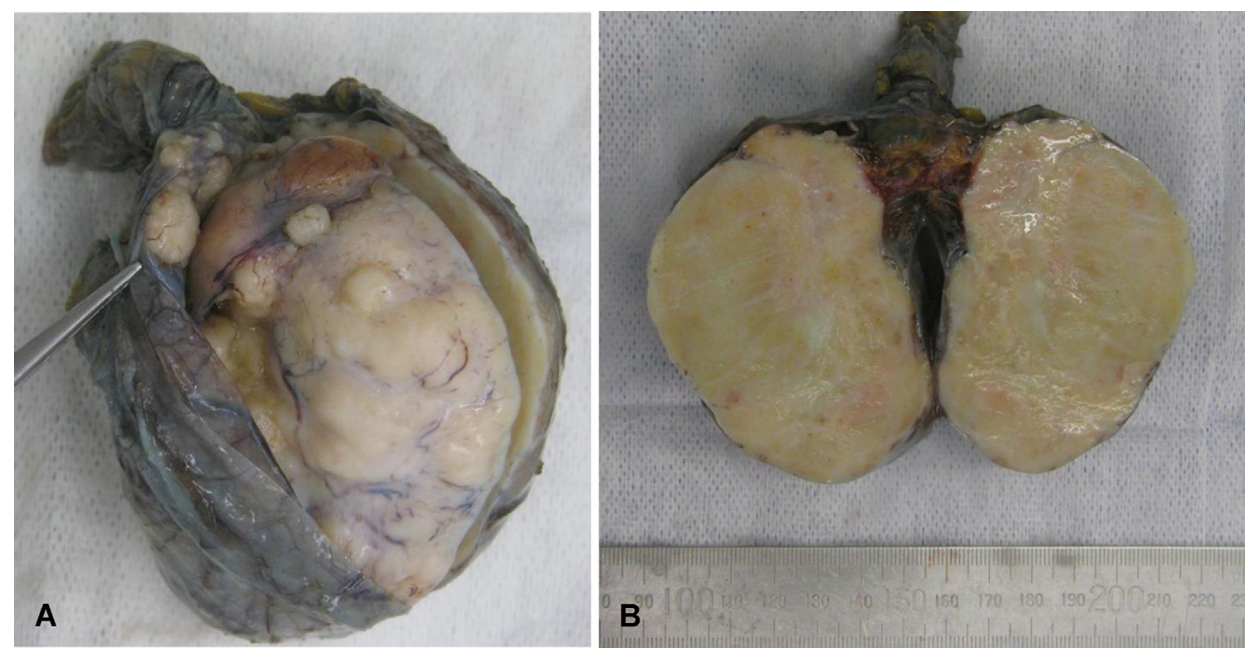

Fig. 1 Right radical orchidectomy specimen. (A) Right orchidectomy specimen with parietal layer of tunica vaginalis divided, demonstrating nodular testicular tumour with satellite nodules on tunica. (B) Bisected specimen demonstrating tan coloured tumour replacing entire contents of testis and epididymis. 

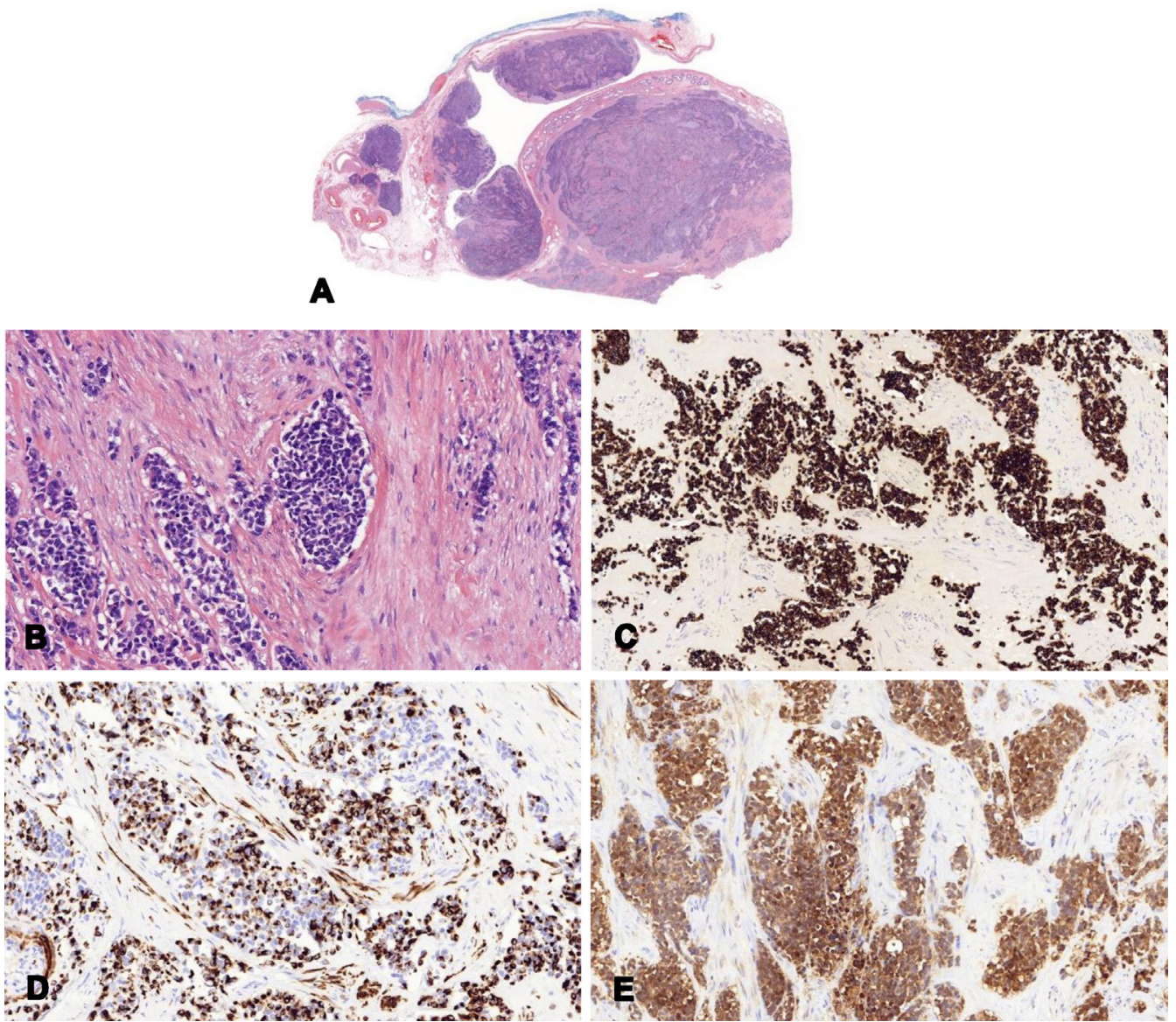

Fig. 2 Microscopic images showing nests of tumour cells surrounded by a characteristic desmoplastic stroma at (A) low power and (B) higher magnification. Immunohistochemistry shows the characteristic multiphenotypic differentiation including (C) Cam5.2, (D) desmin and (E) neuron specific enolase.

enolase (NSE) demonstrating epithelial, muscular, and neural differentiation (Fig. 2). CD99 was also positive and myogenin was negative. WT-1 was negative, however the antibody for the N-terminus was used. The morphology and immunohistochemistry were characteristic for a desmoplastic small round cell tumour and the diagnosis was subsequently confirmed with fluorescence in situ hybridisation for the EWSRl gene rearrangement. Following surgery, he was treated with intensive chemotherapy consisting of vincristine, doxorubicin and cyclophosphamide alternating with ifosfamide and etoposide. He tolerated the first three cycles of chemotherapy well and the most recent imaging demonstrated a slight reduction in intra-abdominal disease.

DSRCT is a rare tumour, with primaries of the testis and paratesticular region even rarer. The largest case series to date includes six cases of primary paratesticular lesions, ${ }^{6}$ with a number of other individual cases reported. ${ }^{7}$ There have been only four published cases of primary testicular DSRCT. ${ }^{2-4}$ In this case, it is impossible to determine the specific origin of the tumour as at the time of imaging and orchidectomy the tumour had replaced the entire contents of the testis. There was a wide range of differential diagnoses for this case, including germ cell tumours, primitive neuroectodermal tumour, poorly differentiated sex cord stromal tumours and clear cell sarcoma. With no evidence of a large abdominal or pelvic mass, the diagnosis of DSRCT was not clinically suspected and histopathological examination was required to make this rare diagnosis. DSRCT is typically a highly malignant tumour with a propensity for involvement of serosal surfaces. ${ }^{8}$ Patients usually present with vague, nonspecific symptoms, such as abdominal pain, bloating, nausea and vomiting. ${ }^{1,9}$ While extra-abdominal metastases are not uncommon, extra-abdominal primary sites remain rare. $^{3}$ Paratesticular DSRCT has been reported more commonly than tumours of testicular origin. ${ }^{7}$ Clinical features of testicular DSRCT appear very similar to other advanced testicular tumours and include an enlarged testicular mass with palpable lymphadenopathy and metastatic disease on imaging. As such, this entity remains difficult to distinguish from other testicular tumours and relies on formal histopathological diagnosis. ${ }^{4}$ On computed tomography imaging, the largest intra-abdominal lesion was a $6 \mathrm{~cm}$ paraaortic mass, and on histopathological analysis the visceral tunica vaginalis was intact. There was no hydrocele seen on imaging to suggest a patent processus vaginalis. Some data from reported cases suggests that tumours originating from the testes tend to invade paratesticular structures, whereas paratesticular primaries do not. ${ }^{4,6,7}$ Therefore, it is likely that this case represents primary testicular DSRCT with metastasis. However, it is possible that this lesion represents a haematogenous metastasis to the testis, direct seeding via a patent processus vaginalis which is now obliterated with tumour, direct spread down the spermatic cord structures or local invasion from a primary paratesticular tumour.

Histopathological analysis of DSRCT typically reveals a nested, small, round, blue cell tumour with high grade 
features surrounded by a desmoplastic stroma. ${ }^{2}$ The immunohistochemical profile of DSRCT shows a characteristic mutliphenotypic differentiation, expressing epithelial, muscular, and neural markers. Classically, these usually manifest as positivity for EMA, pancytokeratins, CD57, NSE and desmin with negative staining for myogenin. ${ }^{8}$ This tumour has a characteristic chromosomal translocation at $\mathrm{t}(11 ; 22)(\mathrm{p} 13 ; \mathrm{q} 12)$ which fuses the EWSR1 gene and Wilms tumour suppressor (WT1) gene..$^{8,10,11}$ This has been shown to upregulate a variety of factors associated with tumourigenesis including epidermal growth factor receptor, interleukin-2 receptor $\beta$ and endogenous platelet derived growth factor. ${ }^{1,8}$ Prognosis is poor, with a reported 5-year survival rate ranging from 15 to $30 \%{ }^{7,10}$ Current treatment modalities include surgical resection, chemotherapy and radiotherapy, while novel approaches are being explored. ${ }^{12}$

We report this very rare sarcoma mimicking a testicular germ cell tumour. Initially, this was an extremely difficult case to manage and diagnose clinically. Ultimately, pathological assessment of the resected specimen, along with cytogenetics, was required to make the diagnosis. The clinical differential diagnosis of testicular masses in a young male very rarely includes this tumour. Histologically, in the peritoneum in a young male, this tumour can be easily recognised but might not be initially thought of, especially with this presentation and the rarity of this entity in this location. This case is also a harrowing tale of the dangers of noncompliance. This is an extremely aggressive tumour, and delays to diagnosis and treatment by even a few months may diminish overall survival.

Acknowledgements: We would like to thank the patient who provided permission to publish this case report.

\section{Oliver Best $^{1,2}$, Michael Brooks ${ }^{1,3}$, Paul Gassner ${ }^{1}$, Tristan Rutland ${ }^{4,5}$}

${ }^{1}$ Department of Urology, Liverpool Hospital, Liverpool, NSW, Australia; ${ }^{2}$ Sydney Medical School, Faculty of Medicine and Health, University of Sydney, Sydney, NSW, Australia; ${ }^{3}$ School of Medicine, University of New South Wales, Randwick, NSW, Australia; ${ }^{4}$ Department of Pathology, Liverpool Hospital, Liverpool, NSW, Australia; ${ }^{5}$ School of Medicine, University of Western Sydney, Sydney, NSW, Australia

Contact Dr Oliver Best.

E-mail: oliver.best@live.com

1. Lal DR, Su WT, Wolden SL, et al. Results of multimodal treatment for desmoplastic small round cell tumors. J Pediatr Surg 2005; 40: $251-5$.

2. He L, Wen S, Hu X, et al. Primary desmoplastic small round cell tumor of the testis: a case report and review of the literature. Oncol Lett 2013; 6: $565-7$.

3. Manjula M. Pawar Y. Primary desmoplastic small round cell tumor of the testis: first case in India and review of the literature. $J$ Cancer Res Ther 2015; 11: 650.

4. Zhang G-M, Zhu Y, Gan H-L, et al. Testicular desmoplastic small round cell tumor: a case report and review of literature. World J Surg Oncol 2014; $12: 1-4$.

5. Miller KD, Nogueira L, Mariotto AB, et al. Cancer treatment and survivorship statistics. CA Cancer J Clin 2019; 69: 363-85.

6. Cummings OW, Ulbright TM, Young RH, et al. Desmoplastic small round cell tumors of the paratesticular region: a report of six cases. Am J Surg Pathol 1997; 21: 219-25.
7. Sedig L, Geiger J, Mody R, et al. Paratesticular desmoplastic small round cell tumors: a case report and review of the literature. Pediatr Blood Cancer 2017; 64: e26631.

8. Thway K, Noujaim J, Zaidi S, et al. Desmoplastic small round cell tumor: pathology, genetics, and potential therapeutic strategies. Int $J$ Surg Pathol 2016; 24: 672-84.

9. Dufresne A, Cassier P, Couraud L, et al. Desmoplastic small round cell tumor: current management and recent findings. Sarcoma 2012; 2012 714986.

10. Honoré C, Delhorme J, Nassif E, et al. Can we cure patients with abdominal desmoplastic small round cell tumor? Results of a retrospective multicentric study on 100 patients. Surg Oncol 2019; 29: $107-12$.

11. Ladanyi M, Gerald W. Fusion of the EWS and WT1 genes in the desmoplastic small round cell tumor. Cancer Res 1994; 54: 2837-40.

12. Hayes-Jordan A, LaQuaglia MP, Modak S. Management of desmoplastic small round cell tumor. Semin Pediatr Surg 2016; 25: 299-304.

DOI: https://doi.org/10.1016/j.pathol.2021.07.010

\section{Desmoplastic small round cell tumour presenting as cervical lymph nodes metastases with solid pattern morphology and novel EWSR1-WT1 fusion transcript}

To the Editor,

Desmoplastic small round cell tumour (DSRCT) is an aggressive, paediatric mesenchymal neoplasm that predominantly involves the abdominal or pelvic peritoneum and often presents with widespread abdominal/peritoneal implants or metastases. Histologically, DSRCT has a characteristic appearance of nests of fairly uniform small round cells setting in variable amounts of desmoplastic stroma and immunohistochemically it exhibits a polyphenotypic multi-directional differentiation, with co-expression of epithelial, neural, and muscle markers. ${ }^{1,2}$ Cytogenetically, DSRCT is characterised by a recurrent $\mathrm{t}(11 ; 22)(\mathrm{p} 13 ; \mathrm{q} 12)$ translocation, leading to formation of the EWSR1-WT1 fusion transcripts that are composed mainly of the first 7 exons of the EWSR 1 gene and the last three exons (exons $8-10$ ) of the WT1 gene. ${ }^{3}$ The diagnosis of DSRCT is relatively straightforward if the tumour arises in typical locations, exhibiting both its characteristic morphological and immunohistochemical features. However, DSRCT can occur more rarely in extra-abdominal/peritoneal sites either as primary or metastatic tumour and can display a broad spectrum of both morphological features and immunoprofiles, which may cause diagnostic confusion with other small round cell tumours. ${ }^{1}$ In these contexts, detection of the EWSR1-WT1 fusion represents a very useful tool for the differential diagnosis of DSRCT from its mimics.

We report a unique case of DSRCT that presented as cervical lymph nodes metastases, which showed solid morphology with minimal or no desmoplastic stroma and harboured a novel EWSRI-WT1 fusion gene detected by targeted next generation sequencing (NGS) with confirmation by fluorescence in situ hybridisation (FISH) studies.

The patient was a 25-year-old, previously healthy Chinese male who presented with enlargement of a left neck mass of 2 months duration. Physical examination identified a $2.0 \mathrm{~cm}$, firm, rubbery, unfixed mass with multiple mobile small nodules in the left anterior upper neck, which were indicated as 